\title{
DIVERSIDAD Y DISTRIBUCION DE LAS ESPECIES DE MALLOPHAGA (INSECTA) EN AVES Y MAMÍFEROS DE LA COMUNIDAD DE MADRID
}

\author{
M. P. Martín Mateo*
}

\begin{abstract}
RESUMEN
Se presenta información general sobre la biodiversidad y distribución de los Mallophaga parásitos de aves y mamíferos en la Comunidad de Madrid. Con base en datos obtenidos en colecciones del MNCN (Madrid) y revisión de la bibliografía de referencia, se obtienen un total de 145 especies de aves y 12 de mamíferos hospedadores potenciales de 306 especies de malófagos en la Comunidad de Madrid. De ésas, solo 29 especies de aves $(20 \%)$ y 2 de mamíferos $(14,3 \%)$ han sido exploradas, las cuales podrían estar parasitadas por, al menos, 91 especies diferentes de malófagos, pero solo se conocen citas de 40 especies (44\%). En cada especie indicada se señalan los hospedadores conocidos, material y referencia correspondiente para cada especie de Mallophaga citada en la Comunidad de Madrid.
\end{abstract}

Palabras clave: Mallophaga, aves, mamíferos, Comunidad de Madrid, España

\section{ABSTRACT \\ Diversity and distribution of species of Mallophaga (Insecta) on birds and mammals from Comunidad de Madrid}

Based on the entomological collection of the Museo Nacional de Ciencias Naturales, MNCN (Madrid, Spain) and the literature, a total of 145 species of birds and 12 species of mammals that could be potential hosts of Mallophaga, are cited for Madrid. These species are potential hosts to 306 species of Mallophaga. Only 29 species of birds (20\%) and 2 species of mammals $(14,3 \%)$ have been searched for the presence of Mallophaga. These species could be potential hosts of 91 different Mallophaga species, but only 40 species (44\%) are recorded. Each species present in Madrid is indicated with its the host, the material known and respective references.

Key words: Mallophaga, birds, mammals, Comunidad de Madrid, Spain

\section{Introducción}

El conocimiento de la diversidad faunística en la Comunidad de Madrid es extraordinariamente limitado y parcialmente enfocado a grupos zoológicos determinados. Otros grupos zoológicos como los insectos, con un gran número de especies conocidas, han sido poco o nada estudiados en la región. Esto hace que el primer paso, básico para el conocimiento de esa biodiversidad biológica, sea cono-

* Museo Nacional de Ciencias Naturales. José Gutierrez Abascal, 2. 28006 MADRID. e-mail: mpmartin@mncn.csic.es 
cer las especies existentes o posibles y donde están, de los diferentes y variados grupos que constituyen el mundo entomológico.

Uno de estos grupos faunísticos son los Mallophaga (malófagos) insectos que viven sobre aves y mamíferos, desarrollando todo su ciclo biológico en el cuerpo del hospedador. Como ectoparásitos obligados están distribuidos por todo el mundo en la mayoría de las familias de aves y así mismo presentes en algunos mamíferos. Su parasitismo determina el interés de su estudio y conocimiento ya que el grado y la intensidad de sus infestaciones pueden influir directamente en la salud de los hospedadores, incluso en caso de infestaciones masivas en la muerte de los mismos. Su especificidad parasitaria les convierte además en referencia para estudios de biodiversidad, siendo un modelo interesante de organismos en estudios de coespeciación (Ridley, 1996; Smith, 2001).

Este trabajo ofrece un panorama actualizado sobre el conocimiento taxonómico de los malófagos en la Comunidad de Madrid, a la vez que recoge una primera aproximación sobre la diversidad y la distribución del grupo de insectos estudiados.

\section{Diversidad de Mallophaga}

Se conocen en el mundo alrededor de 4.500 especies de malófagos, parásitos de aves (3.900 especies) y mamíferos (600 especies). Price et al. (2003) han publicado una Check List que recopila y actualiza todas las listas anteriores (Kellogg, 1908; Harrison, 1916; Hopkins \& Clay, 1952, 1953, 1955; Emerson, 1972). En ella los autores presentan una relación pormenorizada de los malófagos y sus hospedadores, con datos sobre su taxonomía, clasificación, distribución en los órdenes y familias de aves y mamíferos y relaciones parásitohospedador.

Los Mallophaga (piojos masticadores) son insectos Pterigotas con metamorfosis simple o incompleta (hemimetábolos) que, junto con los Anoplura o Siphunculata (piojos chupadores) constituyen el orden Phthiraptera de Haecker, 1896. Ambos se incluyen en el grupo de órdenes Hemipteroideos de la sección Exopterigota (Richard \& Davies, 1977). Los Mallophaga comprenden tres subórdenes: Amblycera, Ischnocera y Rhynchopthirina, siendo los dos primeros los que contienen especies cuyos hospedadores pueden ser encontrados en la Comunidad de Madrid.

Los caracteres morfológicos básicos de las especies de ambos subórdenes son bastante unifor- mes (Price et al., 2003) (Fig. 1) pero superficialmente aparecen características muy diversas especialmente en las especies de Ischnocera que, según Clay (1949), han adquirido ya en su evolución, paralela a la de sus aves hospedadoras, cuando éstas tenían un plumaje más uniforme, antes de su divergencia en diferentes grupos con las consiguientes modificaciones en la estructura de sus plumas. Johnson \& Clayton (2003) ilustran también la diversidad de caracteres en las diferentes familias de malófagos (Fig. 2)

La revisión de la bibliografia mundial sobre Malófagos, muy especialmente la Check List citada (Price et al., 2003) pone de manifiesto las especies de malófagos potenciales que pueden vivir sobre las especies de aves y mamíferos hospedadores considerados en la Comunidad de Madrid, proporcionandonos un conocimiento de la distribucion de estos parásitos en las comunidades de aves y mamíferos.

\section{Material y Métodos}

En la Península Ibérica se conocen unas 250 especies de malófagos parásitos de aves y unas 16 parásitos de mamíferos (Martin Mateo, 2002; Martin Mateo, en preparación) Una primera aproximación al conocimiento de la biodiversidad de los malófagos parásitos de aves y mamíferos de la Comunidad de Madrid se ha obtenido mediante:

$1^{\circ}$ ) La revisión de las especies de aves y mamíferos censados en dicha comunidad

$2^{\circ}$ ) Mediante una exhaustiva consulta de la bibliografia a nivel mundial, la determinación de las especies de malófagos potencialmente posibles sobre las especies de aves y mamíferos censadas en Madrid

$3^{\circ}$ ) Un repaso a las citas bibliográficas sobre malófagos citados en España, que ponen de manifiesto las especies estudiadas en la misma comunidad

$4^{\circ}$ ) Comparación de los datos obtenidos para establecer las carencias del conocimiento de tales insectos en el área geográfica objeto de estudio.

Existen en la Comunidad de Madrid cerca de 300 especies de aves censadas ( Del Moral et al., 2002; De la Puente et al., 2003), con estatus provincial diverso: accidentales, que se presentan con muy poca frecuencia; estivales, habituales en primavera y verano; invernantes, habituales en invierno y otoño; 

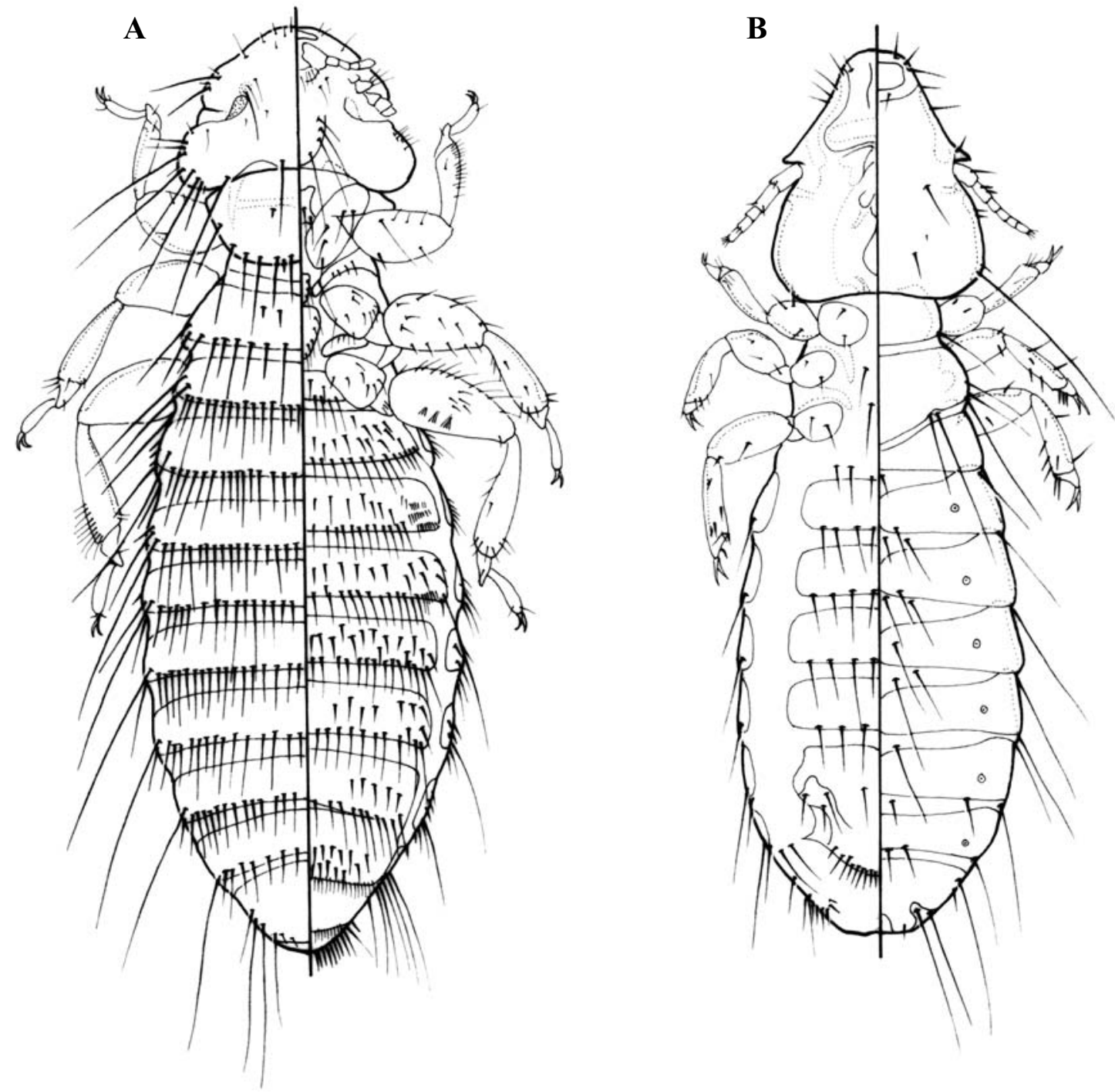

Fig. 1.- Caracteres morfológicos externos de Mallophaga: A) Vista dorsal-ventral de Amblycera; B) Vista ventral-dorsal de Ischnocera.

Fig. 1. - External morphological characters of Mallophaga. A) Dorsal-ventral view of female Amblycera. B) Ventral-dorsal view of female Ischnocera.

migrantes, habituales durante los pasos migratorios (normalmente en primavera y otoño) y sedentarias, habituales a lo largo de todo el año; todas ellas con mayor o menor abundancia de ejemplares.

Considerando las especies que viven sedentarias, con una abundancia variable, en la Comunidad y aquellas estivales, invernantes y migratorias, que aparecen con un número considerable en los períodos de estancia o paso, tendremos una muestra representativa de las aves hospedadoras existentes en el área de estudio objeto de este trabajo. Una lista de las aves consideradas aparece en la Tabla 1.

Una revisión de la bibliografia penínsular de malófagos así como de la Colección del Museo Nacional de Ciencias Naturales proporciona datos acerca de las citas de malófagos estudiados sobre aves localizadas en la Comunidad de Madrid y su distribución en las aves sobre las que han sido colec- 

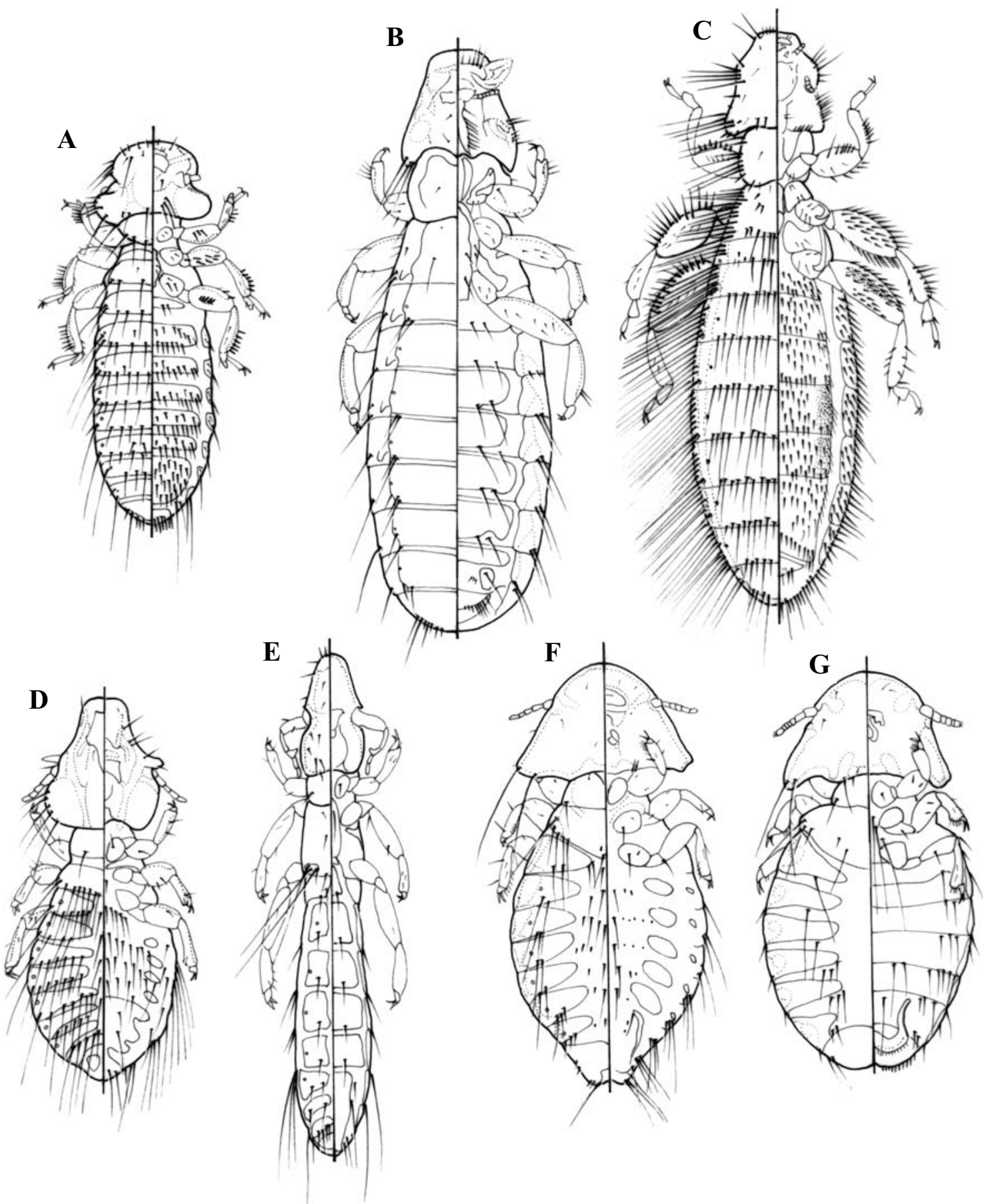

Fig. 2.- Especies representativas de familias de Mallophaga. A) Colpocephalum sp. (Amblycera: Menoponidae); B) Ricinus sp. (Amblycera: Ricinidae); C) Laemobothrion sp. (Amblycera : Laemobothriidae); D) Philopterus sp. (Ischnocera: Philopteridae); E) Columbicola sp. (Ischnocera: Philopteridae); F) Goniodes sp. (Ischnocera: Goniodidae); G) Heptapsogaster sp. (Ischnocera: Heptasogasteridae).

Fig. 2.- Representative species of Mallophaga families. A) Colpocephalum sp. (Amblycera: Menoponidae); B) Ricinus sp. (Amblycera: Ricinidae); C) Laemobothrion sp. (Amblycera: Laemobothriidae); D) Philopterus sp. (Ischnocera: Philopteridae); E) Columbicola sp. (Ischnocera: Philopteridae); F) Goniodes sp. (Ischnocera: Goniodidae); G) Heptapsogaster sp. (Ischnocera: Heptasogasteridae). 
Tabla 1. - Lista de Mallophaga citados sobre aves de la Comunidad de Madrid (las especies estudiadas en Madrid se señalan con un asterisco *).

Table 1.- Check list of Mallophaga recorded on birds of Comunidad de Madrid (species studied in Madrid to mark with an asterisk).

\begin{tabular}{l} 
AVES \\
\hline Tachybaptus ruficollis (Zampullín común) \\
Podiceps cristatus (Somormujo lavanco) \\
Podiceps nigricollis (Zampullín cuellinegro) \\
Phalacrocorax carbo (Cormorán grande)
\end{tabular}

Nycticorax nycticorax (Martinete común) Bubulcus ibis (Garcilla bueyera)

Egretta garcetta (Garceta común)

Ardea cinerea (Garza real)

Ciconia nigra (Cigüeña negra) *

Ciconia ciconia (Cigüeña blanca)

Anser anser (Ánsar común)

Anas strepera (Ánade friso )

Anas crecca (Cerceta común )

Anas platyrrhynchos (Ánade azulón)

Anas clypeata (Cuchara común)

Netta rufina (Pato colorado)

Aythya ferina (Porrón europeo)
MALÓFAGOS

Aquanirmus bahli Pseudomenopon dolium Aquanirmus podicepis Pseudomenopon dolium

Pseudomenopon dolium Eidmaniella pellucida Pectinopygus gyricornis Ciconiphilus decimfasciatu Ardeicola expallidus Ciconiphilus decimfasciatus Ardeicola expallidus Ciconiphilus decimfasciatu. Ardeicola ardeae Ciconiphilus decimfasciatus Ardeicola maculatus Ciconiphilus quadripustulatus

Colpocephalum nigrae

Neophilopterus tricolor * Ardeicola ciconiae

Ciconiphilus quadripustulatus Colpocephalum zebra

Neophilopterus incompletus Anaticola anseris Anatoecus dentatus Anatoecus icterodes

Ciconiphilus pectiniventris Holomenopon leucoxanthum Ornithobius mathisi Trinoton anserinum Anaticola crassicornis Anatoecus dentatus Anatoecus icterodes

Holomenopon clypeilargum Holomenopon leucoxanthum

Holomenopon setigerum Trinoton querquedulae Anaticola crassicornis Anatoecus dentatus Anatoecus icterodes

Holomenopon clypeilargum Holomenopon leucoxanthum Holomenopon stigerum Trinoton querquedulae Anaticola crassicornis Anatoecus dentatus Anatoecus icterodes

Holomenopon leucoxanthum Trinoton querquedulae Anaticola crassicornis Anatoecus dentatus Anatoecus icterodes

Holomenopon clypeilargum Holomenopon leucoxanthum

Holomenopon setigerum

Trinoton querquedulae

Acidoproctus moschatae

Anaticola mergiserrati

Anatoecus dentatus Anatoecus icterodes

Holomenopon leucoxanthum

Trinoton querquedulae

Anaticola mergiserrati

Anatoecus dentatus

Anatoecus icterodes

Holomenopon leucoxanthum

Trinoton querquedulae

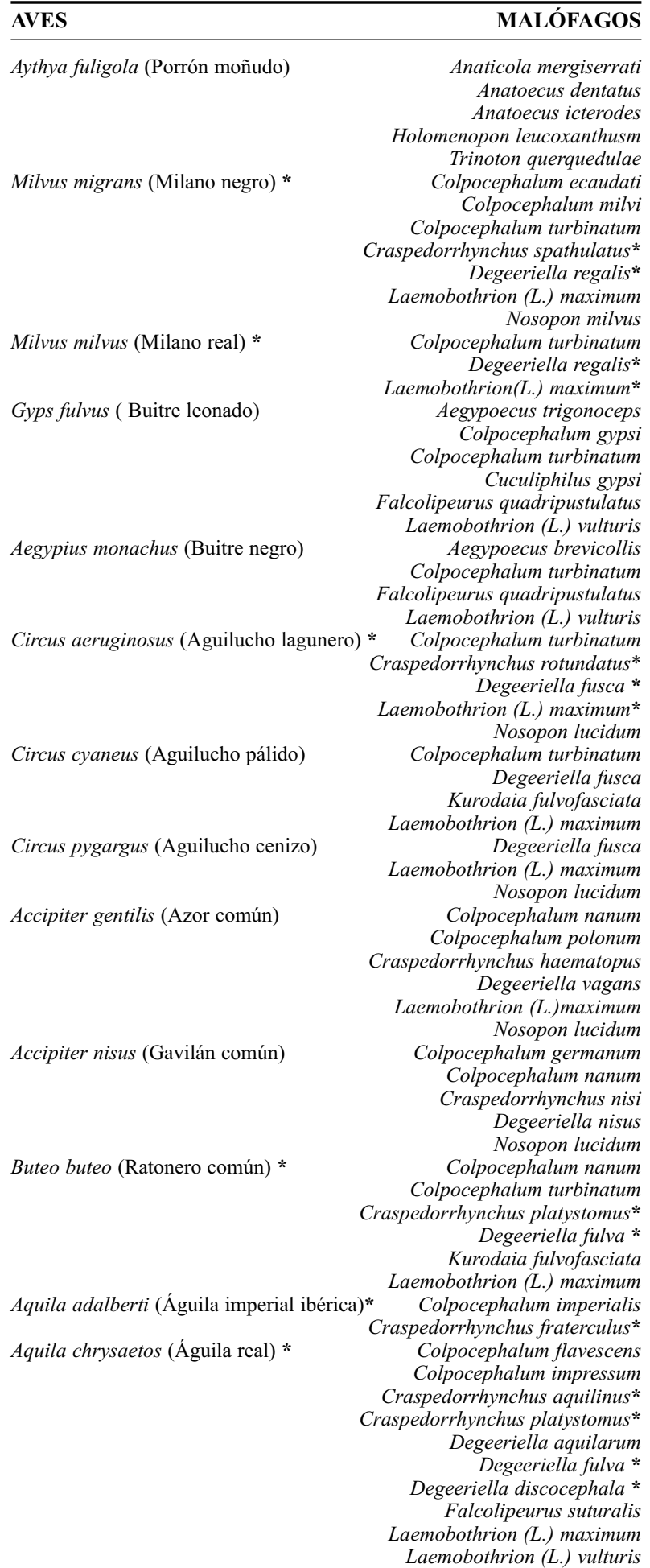




AVES
Hieraaetus pennatus (Águila calzada)

Hieraaetus fasciatus (Águila pedicera) *

Falco tinnunculus (Cernícalo vulgar)*

Falco subbuteo (Alcotán)*

Falco peregrinus (Halcón peregrino)

Alectoris rufa (Perdiz roja)

Coturnix coturnix (Codorniz)

Rallus acuaticus (Rascón europeo)

Gallinula chloropus (Gallineta común)

Porphyrio porphyrio (Calamón común)

Fulica atra (Focha común)

Grus grus (Grulla común) *

Tetrax tetrax (Sisón común)

Otis tarda (Avutarda)

Himantopus himantopus (Cigueñuela )

Burhinus eodicnemus (Alcaraván)

Vanellus vanellus (Avefría ) *

Gallinago gallinago (Agachadiza común)*

Tringa ochropus (Andarríos grande )
MALÓFAGOS

Colpocephalum milvi Colpocephalum turbinatum Craspedorrhynchus ranjhae Degeeriella fulva*

Falcolipeurus jasminae

Laemobothrion (L.) maximum Craspedorrhynchus fasciati Degeeriella fulva *

Kurodaia fulvofasciatsa

Laemobothrion (L.) maximum Colpocephalum subzerafae Degeeriella rufa *

Laemobothrion (L.) tinnunculi Nosopon lucidum Craspedorrhynchus subbuteonis Degeeriella rufa*

Laemobothrion tinnunculi Colpocephalum subzerafae Colpocephalum zerafae Degeeriella rufa

Laemobothrion (L.) tinnunculi Nosopon lucidum Amyrsidea perdicis Cuclotogaster obscurior Goniocotes obscurus Goniodes dispar Goniodes graecus Menacanthus lyal Menopon pallens

Amyrsidea fulvomaculata

Cuclotogaster cinereus

Goniodes astrocephalus Menacanthus abdominalis Fulicoffula rallina Incidofrons ralli Pseudomenopon scopulacorne Rallicola cuspidatus Incidofrons gallinulae Laemobothrion(E.) chloropodis Pseudomenopon pilosum Rallicola minutus

Pseudomenopon concretum Rallicola lugens Rallicola thompsoni Fulicoffula lurida Incidofrons fulicae Laemobothrion (E.) atrum

Pseudomenopon pilosum Rallicola fulicae

Esthiopterum gruis * Gruimenopon longum *

Heleonomus macilentus

Saemundssonia integer Otidoecus antilogus Otidoecus turmalis

Colpocephalum benoiti Pseudomenopon cinereum Rallicola flevescens Actornithophilus tetralicis

Quadraceps annulatus Actornithophilus gracilis Austromenopon vanelli * Lunaceps holophaeus Quadraceps junceus Saemundssonia platygaster Austromenopon durisetosum

Cummingsiella ambigua*

Rhynonirmus scolopacis

Saemundssonia kratochvili Actornithophilus lyallpurensis Austromenopon sohni Quadraceps ochropi

Saemundssonia platygaster

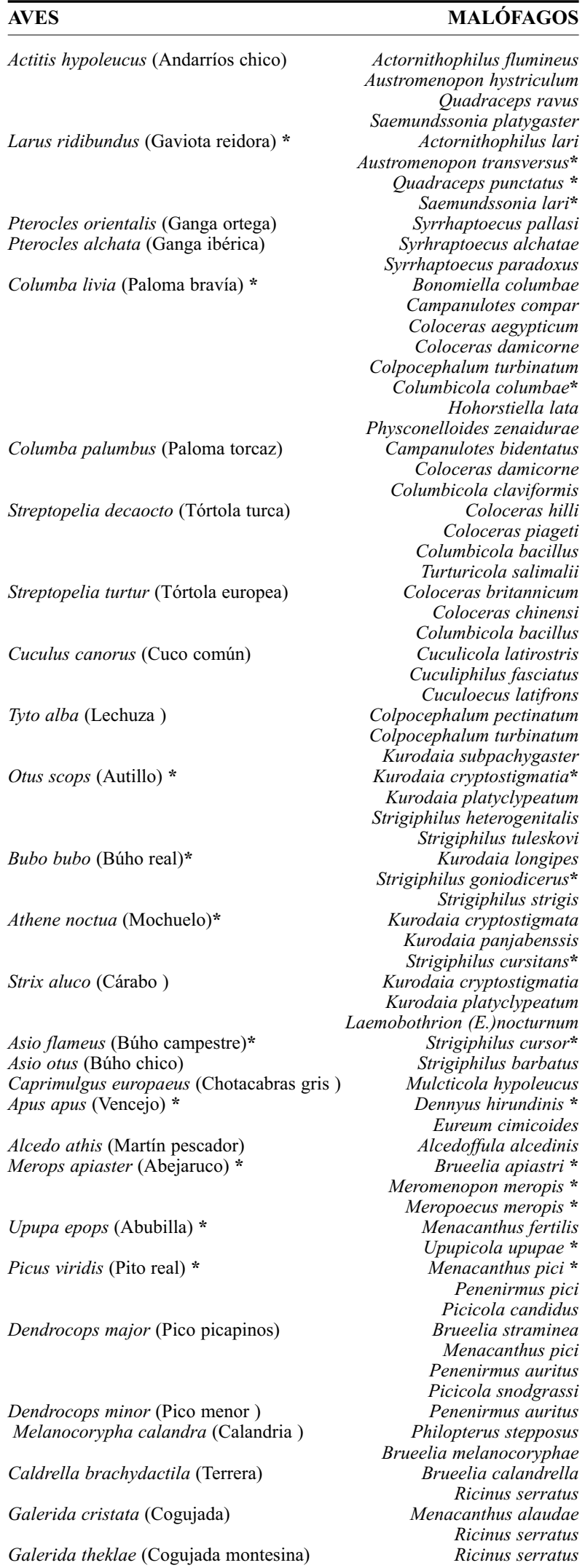




AVES
Lullula arborea (Totovía)
Alauda arvensis (Alondra)
Ptyonoprogne rupestris (Avión roquero)
Hirtundo rustica (Golondrina)

Hirtundo rustica (Golondrina)

\section{Delinchon urbica (Avión común)}

Anthus pratensis (Bisbita común)

Anthus spinoletta (Bisbita alpina)

Motacilla flava (Lavandera boyera)

Motacilla alba (Lavandera blanca)

Cinclus cinclus (Mirlo acuático)

Troglodytes troglodytes (Chochín)

Prunella modularis (Acentor común)

Erithacus rubecula (Petirrojo)

Luscinia megarrhynchos (Ruiseñor común) Luscinia svecica (Pechiazul) Phoenicurus ocrurus (Colirrojo tizón ) Saxicola torquata (Tarabilla común) Oenanthe oenanthe (Collalba gris)

Monticola solitarius (Roquero solitario) Turdus merula (Mirlo común) *

Turdus philomelus (Zorzal común)

Turdus viscivorus (Zorzal charlo )

Cettia cetti (Ruiseñor bastardo) Acrocephalus scirpaceus ( Carricero común)*

Acrocephalus arundinaceus (Carricero tordal) Sylvia communis (Curruca zarcera) Sylvia borin (Curruca mosquitera)

Sylvia atricapilla (Curruca capirotada)

Phylloscopus bonelli (Mosquitero papialbo) Phylloscopus collybita (Mosquitero común)
MALÓFAGOS

Philopterus arboreus Brueelia lulullae

Menacanthus alaudae

Penenirmus hibari

Ricinus serratus Brueelia buettikeri

Machaerilaemus malleus Myrsidea rustica

Philopterus microsomaticus Bruelia gracilis

Philopterus excisus

Brueelia corydalla

Menacanthus pusillus

Ricinus fringillae

Brueelia corydalla

Menacanthus pusillus

Ricinus fringillae

Brueelia kratochvili

Menacanthus pusillus

Philopterus pavidus

Menacanthus nogoma

Menacanthus pusillus

Philopterus passerinus Ricinus fringillae Philopterus cincli

Myrsidea franciscoloi

Menacanthus tenuifrons Myrsidea troglodyti

Penenirmus albiventris

Philopterus trogrlodytis Brueelia modularis

Menacanthus eurysternus

Philopterus modulari Ricinus elongatus Ricinus rubeculae Brueelia tristis

Philopterus rubeculae Ricinus rubeculae Brueelia lais Ricinus rubeculae Ricinus rubeculae

Sturnidoecus irritans

Menacanthus affinis

Menacanthus exilis

Philopterus oenanthe Ricinus rabeculae Brueelia jacobi

Brueelia merulensis

Menacanthus eurysternus

Myrsidea thoracica

Philopterus turdi*

Ricinus elongatus *

Brueelia turdinulae

Menacanthus eurysternus

Philopterus turdi

Ricinus elongatus

Brueelia biscivori

Menacanthus eurysternus

Myrsidea thoracica

Philopterus vernu.

Ricinus elongatus

Menacanthus takayamei Menacanthus eisenachensis

Philopterus necopinatus *

Menacanthus currucae

Menacanthus currucae Brueelia borini

Menacanthus currucae Brueelia neotricapillae Brueelia paratricapillae

Menacanthus currucae

Philopterus phyloscop

Menacanthus agilis

AVES MALÓFAGOS

Phylloscopus trochilus (Mosquitero musical)

Menacanthus agilis Menacanthus currucae Philopterus phyloscopi Penenirmus phyloscopi

Philopterus reguli Ricinus frenatus

Regulus regulus (Reyezuelo sencillo)

Muscicapa striata (Papamoscas gris )

Ficedula hypoleuca (Papamoscas corrojillo)

Aegithalus caudatus (Mito)

Parus cristatus (Herrerillo capuchino)

Parus ater (Carbonero garrapinos)

Parus caeruleus (Herrerillo común)

Parus major (Corbonero común) *

Sitta europaea ( Trepador azul)

Certhia brachydactyla (Agateador común)

Remiz pendulinus (Pájaro moscón)

Oriolus oriolus (Oropéndola )

Lanius meridionalis (Alcaudón real)

Lanius senator (Alcaudón común)

Garrulus glandarius (Arrendajo)

Cyanopica cyana (Rabilargo)

Pica pica (Urraca) *

Pyrrhocorax pyrrhocorax (Chova piquirroja)

Corvus monedula (Grajilla)

Corvus corone (Corneja negra)

Corvus corax (Cuervo) *

Sturnus vulgaris (Estornino pinto)

Passer domesticus (Gorrión común)

Passer hispaniolensis (Gorrión moruno)
Menacanthus agilis
Philopterus capillatus

Penenirmus pari

Sturnidoecus quadrilineatus Ricinus fringillae

Menacanthus sinuatus

Penenirmus pari

Ricinus fringillae

Menacanthus sinuatus

Penenirmus pari

Ricinus fringillae

Menacanthus sinuatus

Philopterus pallescens *

Menacanthus eurysternus Philopterus sittae

Philopterus lineatus Brueelia balati

Menacanthus eurysternus Brueelia munda

Philopterus ornatus

Ricinus dolicocephalus

Brueelia imponderabilica

Menacanthus camelinus

Philopterus coarctatus

Menacanthus camelinus

Philopterus trigonophorus Brueelia glandari

Menacanthus eurysternus Myrsidea indivisa Philopterus garruli Brueelia cyanea

Colpocephalum fregili

Menacanthus eurysternus

Philopterus martinezi

Menacanthus eurysternus* Myrsidea picae* Philopterus picae*

Colpocephalum fregili

Menacanthus gonophaeus Philopterus dumani Brueelia varia

Menacanthus eurysternus

Menacanthus gonophaeus Myrsidea anathorax

Philopterus guttatus Brueelia uncinosa

Colpocephalum fregili

Menacanthus gonophaeus

Philopterus ocellatus

Brueelia biocellata

Colpocephalum fregili

Menacanthus gonophaeus

Philopterus corvi *

Philopterus osborni

Brueelia nebulosa

Menacanthus eurysternus

Myrsidea cucullaris

Ricinus elongatus

Sturnidoecus sturni

Brueelia subtilis

Menacanthus eurysternus

Myrsidea quadrifasciata

Philopterus fringillae Ricinus fringillae

Philopterus hispaniolensis Sturnidoecua boeevi 


\begin{tabular}{|c|c|c|c|}
\hline AVES & MALÓFAGOS & AVES & MALÓFAGOS \\
\hline \multirow[t]{2}{*}{ Passer montanus (Gorrión molinero) } & $\begin{array}{r}\text { Brueelia cyclothorax } \\
\text { Brueelia subtilis } \\
\text { Myrsidea balati }\end{array}$ & Carduelis cannabina (Pardillo común) & $\begin{array}{r}\text { Brueelia stadleri } \\
\text { Menacanthus alaudae } \\
\text { Ricinus fringillae }\end{array}$ \\
\hline & $\begin{array}{r}\text { Ricinus fringillae } \\
\text { Sturnidoecus ruficeps }\end{array}$ & Loxia curvirostra (Piquituerto común) & $\begin{array}{r}\text { Brueelia limbata } \\
\text { Philopterus curvirostrae }\end{array}$ \\
\hline Petronia petronia (Gorrión chillón) & Philopterus petrinus & Coccothraustes coccothraustes (Picogordo) & Brueelia juno \\
\hline Fringilla coelebs (Pinzon vulgar) & $\begin{array}{r}\text { Menacanthus eurysternus } \\
\text { Ricinus fringillae }\end{array}$ & Emberiza cia (Escribano montesino) & $\begin{array}{r}\text { Ricinus fringillae } \\
\text { Ricinus serratus }\end{array}$ \\
\hline Serinus serinus (Verdecillo) & $\begin{array}{r}\text { Brueelia sexytanum } \\
\text { Myrsidea serini }\end{array}$ & Emberiza hortulana ( Escribano hortelano) & Penenirmus buresi \\
\hline Carduelis chloris (Verderón comúnun) & $\begin{array}{r}\text { Menacantus eurysternus } \\
\text { Myrsidea serini }\end{array}$ & Emberiza schoeniclus (Escribano palustre) & $\begin{array}{r}\text { Brueelia blagovescenskyi } \\
\text { Ricinus fringillae }\end{array}$ \\
\hline Carduelis spinus (Lúgano) & $\begin{array}{c}\text { Philopterus citrinellae } \\
\text { Brueelia chrysomytris }\end{array}$ & Miliaria calandra (Triguero) & $\begin{array}{r}\text { Philopterus cumulatus } \\
\text { Ricinus major }\end{array}$ \\
\hline
\end{tabular}

tados. Estos datos se ponen de manifiesto en la Tabla 1 mediante un asterisco en cada especie correspondiente y así mismo explicitados en el Apéndice.

Las consideraciones expuestas son aplicables también a los malófagos que viven sobre especies de mamíferos, aunque en menor número de hospedadores y con cifras de permanencia considerablemente menores.

Siguiendo el tratamiento dado para el estudio de los malófagos de aves, es decir mediante revisión bibliográfica mundial se pueden conocer las especies de malófagos petencialmente parásitas de mamíferos y mediante revisión de la bibliografia peninsular conocer las citas referidas a la Comunidad de Madrid, lo que nos permitirá hacer las comparaciones pertinentes. Los datos a los que se hace referencia aparecen en la Tabla 2.

\section{Resultados}

En la Comunidad de Madrid se han citado un total de 89 especies de Mallophaga en un total de 29 especies de aves examinadas (Tabla 1 y Apéndice).

Los mamíferos son hospedadores de una sola familia de malófagos, Trichodectidae, incluida en el subórden Ischnocera. Se conocen alrdedor de 400 especies válidas (Price et al., 2003) de las que 26 (incluyendo las cinegéticas) han sido citadas de la Comunidad de Madrid (Blanco \& Gonzalez, 1992; Palomo et al., 2002). De éstas, 12 especies son potencialmente parasitadas por malófagos, el resto lo son por especies de Anoplura, insectos chupadores característicos de mamíferos.

Tabla 2. - Lista de Mallophaga citados sobre mamíferos de la Comunidad de Madrid. (Las especies estudiadas en Madrid se señalan con un asterisco).

Table 2.- Check list of Mallophaga recorded on mammals of Comunidad de Madrid (species studied in Madrid are marked with an asterisk).

\begin{tabular}{ll}
\hline MAMIFEROS & MALÓFAGOS \\
\hline Cervus elaphus (Ciervo común) & Bovicola longicornis \\
Dama dama (Gamo) & Bovicola tibialis \\
Vulpes vulpes (Zorro) & Felicola vulpes \\
Felis silvestris (Gato montés) & Felicola hercinianus \\
Lynx pardinus (Lince) & Felicola isidoroi \\
Lutra lutra (Nutria) & Lutridia exilis \\
Martes foina (Garduña) & Stachiela retusa \\
Meles meles (Tejón)* & Trichodectes melis * \\
Mustela nivalis (Comadreja) & Stachiela mustelae \\
Mustela putorius (Turón) & Stachiela jacobi \\
Genetta genetta (Gineta)* & Felicola genettae * \\
Lepus granatensis (Liebre ibérica) & Procavicola lindfieldi \\
& Procavicola sternatus \\
& Procaviphilus serraticus \\
\hline
\end{tabular}




\section{Conclusiones}

Del análisis de la Tabla 1 podemos concluir:

A) que en las 145 especies de aves que tienen presencia considerable en el área geográfica que constituye la Comunidad de Madrid, pueden vivir potencialmente 292 especies de malófagos;

B) se han explorado para la detección de Mallophaga sólo 29 especies de aves, lo que constituye el $20 \%$;

C) estas 29 especies exploradas, podrían estar parasitadas por 89 especies diferentes de malófagos, de acuerdo con las citas bibliográficas conocidas a nivel mundial;

D) se conocen 38 especies de malófagos citadas sobre aves de la Comunidad de Madrid, lo que constituye un $42,7 \%$ de las especies posibles. (Apéndice).

Conclusiones semejantes se obtienen del análisis de la Tabla 2 : 12 especies de mamíferos suceptibles de ser parasitados por Malófagos viven en la Comunidad de Madrid, hospedadores potenciales de 14 especies de malófagos; solo dos han sido objeto de estudio $(16,6 \%)$ determinandose dos únicas especies de malófagos (14,2\%).

Esta conclusiones dan una idea clara de la escasez del conocimiento de la fauna referida a Mallophaga, así como de su diversidad y distribución, en la Comunidad de Madrid

\section{AGRADECIMIENTOS}

La autora quiere expresar su agradecimiento a la Comunidad de Madrid que ha subvencionado el Proyecto de Investigación GR/AMB/0750/2004. Igualmente agradece a. M. García París su valiosa ayuda y sugerencias que han contribuido a mejorar el trabajo y a L.M. Carrascal de la Puente los datos proporcionados sobre las aves de la Comunidad de Madrid.

\section{Referencias}

Blanco, J. C. \& González, J. L. (eds.), 1992. Libro Rojo de los Vertebrados de España. Ministerio de Agricultura, Pesca y Alimentación (ICONA). Madrid. 714 pp.

Clay, T., 1949. Some problems in the evolution of a group of ectoparasites. Evolution, 3(4): 279-299.

De la Puente, J., Pérez-Tris, J. \& Bermejo, A. (eds.), 2003. Anuario Ornitológico de Madrid 2002. SEOMontícola. Madrid 203 pp.
Del Moral, J. C., Molina, B., De la Puente, J. \& PÉrez-Tris, J., (eds.), 2002. Atlas de las Aves invernantes de Madrid, 1999-2001. Seo-Montícola y Comunidad de Madrid. Madrid. 397 pp.

EMERSON, K. C., 1972a. Checklist of the Mallophaga of North América (North of Mexico) .Part. I. Suborder Amblycera. Deseret Test Center. Dugway. 118 pp.

EMERSON, K. C., 1972b. Checklist of the Mallophaga of North América (North of Mexico). Part.II.Suborder Ischnocera. Deseret Test Center. Dugway. 200 pp.

EMERSON, K. C., 1972c. Checklist of the Mallophaga of North América (North of Mexico). Part. IV. Bird host list. Deseret Test Center. Duway. 216 pp.

Gállego, J., Martín Mateo, M. P. \& Aguirre, J. M., 1987. Malófagos de rapaces españolas. II. Las especies del género Craspedorrhynchus Keler, 1939 parásitas de Falconiformes con descripción de tres especies nuevas .Eos, 63: 31-66

HARrison, L., 1916. The genera and species of Mallophaga. Parasitology, 9 : 1-156

Hopkins, G. H. E. \& Clay, T., 1952. Check List of the genera and species of Mallophaga. British Museum (Natural History). London. 362 pp.

HoPkins, G. H. E. \& Clay, T., 1953. Additions and corrections to the check list of Mallophaga. Annals and Magazine of Natural History, (12), 6: 434-448.

Hopkins, G. H. E. \& Clay, T., 1955. Additions and corrections to the check list of Mallophaga. II. Annals and Magazine of Natural History, (12), 8: 177-190.

Johnson, K. H. \& Clayton, D. H., 2003. The biology, ecology and evolution of chewing lice. In: R. D. Price, R. A. Hellenthal, R. L. Palma, K. P. Johnson and D. H. Clayton. The chewing lice: world checklist ond biological overview. Illinois Natural History Survey Special Publication. Illinois: 449-476.

KellogG, V. L., 1908. Mallophaga. In: Wytsman, Genera Insectorum, 66: 1-87.

Lanzarot, M. P., Martín Mateo, M. P., Merino, S. \& FERNÁNDEZ GARCÍA, M, 2005. A redescription of Neophilopterus tricolor (Burmeister,1938) (Insecta: Phthiraptera: Ischnocera: Philopteridae) from the black stork Ciconia nigra (L.) (Aves) with notes on its prevalence. Journal of Natural History, 39(39): 3471-3480.

MARTín, M. P., 1975. Notas sobre las especies del género Laemobothrion encontradas sobre aves españolas (Mallophaga: Laemobothriidae). Graellsia, [1974], 30: 163-172,

Martín, M. P. y Jiménez Millán, F., 1967. Contribución al conocimiento de las especies de malófagos existentes en España. Graellsia, 23: 143-158.

Martín Mateo, M. P., 1977. Especies de Ricinus (Mallophaga, Insecta) ectoparásitos de aves en España. Revista Ibérica de Parasitología, 37(1-2): 109-122. 
Martín Mateo, M. P., 1978. Malófagos parásitos de Coraciformes. Revisto Ibérica de Parasitología, 38(1-2): 385-402.

Martín Mateo, M. P., 1984. Estudio taxonómico y biométrico de malófagos parásitos de Picus viridis (L.) Boletin de la Asociación española de Entomología, 8: 151-164.

Martín MateO, M. P., 1988. Malófagos parásitos de aves. Scolopaceps ambiguus (Burmeister, 1838) género y especie nuevos para la fauna ibérica (Philopteridae, Mallophaga). Boletín de la Asociación Española de Entomología, 12: 153-163.

Martín Mateo, M. P., 1989. Estado actual del conociminto de los malófagos (Insecta) parásitos de aves y mamíferos en España. Revista Ibérica de Parasitología, 49(4): 387-410.

Martín Mateo, M. P., 2002. Mallophaga Amblycera. En: Fauna Ibérica, vol 20. Ramos, M. A. et al. (Eds.) Museo Nacional de Ciencias Naturales (CSIC). Madrid. $187 \mathrm{pp}$.
Martín Mateo, M. P. (en preparación). Mallophaga. Ischnocera. En: Fauna Ibérica.

Martín Mateo, M. P. \& GonzÁlez AndúJar, J. L., 1983. Malófagos parásitos de Buteo buteo (L.). Eos, 59: 101-107.

Martín Mateo, M. P. \& Jiménez Millán, F., 1972. Especies españolas del género Colpocephalum (Mallophaga). Cuadernos de Ciencias Biológicas, 2: 53-57.

Palomo, L. J. \& Gisbert, J. (eds.), 2002. Atlas de los mamíferos terrestres de España. Dirección General de Conservación de la Naturaleza y SECEM-SECEMU. Madrid. 564 pp.

Price, R. D., Hellenthal, R.A., Palma, R. L., Johnson, K. P. \& Clayton, D. H., 2003. The chewing lice: world check list and biological overview. Illinois Natural History Survey Special Publication 24. Illinois. $\mathrm{x}+501 \mathrm{pp}$. 
Apendice.- Datos faunísticos. Registros conocidos de las especies de Mallophaga (Insecta) ectoparásitos de aves y mamíferos de la Comunidad de Madrid.

Appendix.- Faunistic data. Records of species of Mallophaga (Insecta) ectoparasites on birds and mammals from Comunidad de Madrid.

\author{
Austromenopon vanelli Zlotorzycka, 1968 \\ FAMILIA: Ancistronidae \\ HOSPEDADOR: Vanellus vanellus (L.) \\ MATERIAL: 1 macho, El Pardo, 9-XI-1934 \\ (Quirós, leg.) Colección MNCN. \\ REFERENCIA: Martín Mateo, 2002.
}

Austromenopon transversum (Denny, 1842)

FAMILIA: Ancistronidae

HOSPEDADOR: Larus sp

MATERIAL: 1 hembra, Madrid, XII-1904

(Martínez de la Escalera, leg.). Colección MNCN

REFERENCIA: Martín y Jiménez Millán, 1967. (Citada como Menopon ridibundum)

Brueelia apiastri (Denny, 1842)

FAMILIA: Philopteridae

HOSPEDADOR: Merops apiaster L.

MATERIAL: 1 hembra, Fuencarral, II-1921 (B. de Quirós, leg.) Colección MNCN

REFERENCIA: Martín y Jiménez Millán 1967

(Citada como Degeeriella apiastri)

Columbicola columbae (Linnaeus, 1758)

FAMILIA: Esthiopteridae

HOSPEDADOR: Columba livia L.

MATERIAL: 1 macho, Navalcarnero, 1957

(Jiménez Millán, leg.) Colección MNCN

REFERENCA: Martín y Jiménez Millán 1967.

Craspedorrhynchus aquilinus (Denny, 1842)

FAMILIA: Philopteridae

HOSPEDADOR: Aquila chrysaetos (L.)

MATERIAL: 7 machos, 10 hembras, 1 ninfa,

24-XI-1982 (Aguirre,leg.) Madrid (Casa de Campo). Colección Aguirre, MNCN

REFERENCIA: Gállego, Martín Mateo y Aguirre, 1987

Craspedorrhynchus fraterculus Eichler y Zlotorzycka, 1972

FAMILIA: Philopteridae

HOSPEDADOR: Aquila heliaca Svigny

MATERIAL: 10 machos, 18 hembras, 8 ninfas, 4-III-1983 (Aguirre, leg). Brunete. Colección Aguirre, MNCN

REFERENCIA: Gállego et al., 1987.

Craspedorrhynchus platystomus (Burmeister, 1838)

FAMILIA: Philopteridae

HOSPEDADOR: Buteo buteo (L.) y Aquila sp.

MATERIAL: 1 macho y 1 hembra, XI- 1904 (Martínez de la Escalera, leg.) ; 1 macho , 1 hembra, X-1904 (Martínez de la Escalera, leg.) Madrid. Colección MNCN

REFERENCIA: Martín y Jiménez Millán 1967. (Citada como Philopterus platystomus).
Craspedorrhynchus rotundatus (Piegt, 1880)

FAMILIA: Philopteridae

HOSPEDADOR: Circus aeruginosus (L.)

MATERIAL: 2 machos, 1 hembra, 5-XI-1904 (Martínez de la Escalera, leg.) Madrid; 1 macho, 2 hembras, 1 ninfa, 12-XII-1985 (Aguirre, leg.) Manzanares el Real. Colección MNCN

REFERENCIA: Gállego et al.,1987.

Craspedorrhynchus spathulatus (Giebel, 1874)

FAMILIA: Philopteridae

HOSPEDADOR: Milvus migrans (Boddaert)

MATERIAL: 8 machos, 7 hembras, 1 ninfa, 12-

V-1983 (Aguirre, leg.) Madrid (Casa de Campo), 1 macho, 1 hembra, 18-VI-1984, (Aguirre, leg.) El Molar. Colección Aguirre MNCN

REFERNCIA: Gállego et al.,1987.

Cummingsiella ambigua (Burmeister, 1838)

FAMILIA: Rallicolidae

HOSPEDADOR: Capella gallinago (L.)

MATERIAL: 2 machos, 1 hembra, aunque la etiqueta no indica la localidad, los datos del colector y la fecha hacen suponer que podría ser El Pardo (Madrid), 9-III-1934 ( B.de Quirós leg.) Colección MNCN.

REFERENCIA: Martín Mateo,1988. (Citada como Scolopaceps ambiguus)

Degeeriella discocephala (Burmeister, 1838)

FAMILIA : Degeeriellidae

HOSPEDADOR: "aguila"

MATERIAL: 1 macho, 4 hembras, 9 ninfas, Pozuelo de Alarcón, VI-1058 (Ruano leg.) Colección MNCN

REFERENCIA: Martín y Jiménez Millán, 1967.

Degeeriella fulva (Giebel, 1874)

FAMILIA: Degeeriellidae

HOSPEDADOR: Aquila chrysaetos (L.), Buteo buteo (L.), Hieraaetus pennatus (Gmelin), Hieraaetus fasciatus (Vieillot)

MATERIAL: 1 macho, 4 hembras, 9 ninfas, Pozuelo de Alarcón, VI-1958 (Ruano,leg.); 1macho, 1 hembra, Madrid, X-1904 (Martínez de la Escalera, leg.); 3 machos, 5 hembras, Los Molinos; 14 machos, 10 hembras, Madrid (Casa de Campo), 1983 (Gállego, leg.); 7 machos, 2 hembras, Collado Villalba, 1984 Gállego, leg.); 4 machos, 9 hembras, 2 ninfas, Robledo de Chavela, 1985 (Gállego, leg.); 1 hembra, Aranjuez (Gállego, leg.)

Colección MNCN.

REFERENCIA: Martín y Jiménez Millán, 1967. (Citadas como Degeeriella discocephala y Degeeriella fusca). Martín Mateo y González Andújar, 1983.
Degeeriella fusca (Denny, 1842)

FAMILIA: Degeeriellidae

HOSPEDADOR: Circus aeruginosus (L.)

MATERIAL: 3 hembras, Madrid, XII- 1923 (García Llorens, leg.); 4 machos, 4 hembras, Torrelodones, 1984 (Gállego, leg.); 2 machos, 2 hembras, Alcobendas, 1984 (Gállego, leg.). Colección MNCN

REFERENCIA: Martín y Jiménez Millán, 1967.

Degeeriella regalis (Giebel, 1886)

FAMILIA: Degeeriellidae

HOSPEDADOR: Milvus milvus (L.) y Milvus migrans (Bodd.)

MATERIAL: 4 machos, 4 hembras, Madrid (Casa de Campo), 1983, Gállego, leg.); 1 hembra, El Escorial, 1984 (Gállego, leg.); 1 macho ,1 hembra, El Molar, 1984 (Gállego, leg.). Colección MNCN

Degeeriella rufa (Burmeister, 1838)

FAMILIA: Degeeriellidae

HOSPEDADOR: Falco tinnunculus L. y Falco subbuteo L.

MATERIAL: 1 macho , 1 hembra, San Martín de Valdeiglesias, 1983, (Gállego, leg.) ; 1 macho , Fresnedillas, 1984(Gállego, leg.); 1 hembra, Arganda, 1984 (Gállego, leg.) ; 1 macho, 1 hembra, Torrejón de Velasco, 1985 (Gállego, leg.); 1 hembra, Madrid , 1904 (Martínez de la Escalera, leg.); 1 hembra, Villaconejos, 1984 (Gállego, leg.) Colección MNCN

REFERENCIA: Martín y Jiménez Millán, 1967.

Dennyus hirundinis (Linnaeus, 1761)

FAMILIA: Menoponidae

HOSPEDADOR: Apus apus (L.)

MATERIAL: 1 macho, 1 hembra, Madrid (Ciudad Universitaria) (París, leg.). Colección MNCN

REFERENCIA: Martín Mateo, 2002.

Esthiopterum gruis Harrison, 1916

FAMILIA: Esthiopteridae

HOSPEDADOR: Grus grus (L.)

MATERIAL: 2 machos, 4 hembras, Pozuelo de Alarcón, XII-1958 (Ruano, leg)

REFERENCIA: Martín y Jiménez Millán, 1967.

Gruimenopon longun (Giebel, 1874)

FAMILIA: Ancistronidae

HOSPEDADOR : Grus grus (L.)

MATERIAL: 11 machos, 9 hembras, Pozuelo de Alarcón, 1958 (Ruano, leg.) Colección MNCN

REFERENCIA: Martín Mateo y Jiménez Millán, 1972. (Citada como Colpocephalum atrofasciatum). 
Kurodaia cryptostigmata (Nitzsch, 1861)

FAMILIA: Colpocephalidae HOSPEDADOR: Otus scops (L.)

MATERIAL: 2 machos, 4 hembras, Madrid, IX-1924 (Benedito, leg.) Colección MNCN

REFERENCIA: Martín Mateo y Jiménez Millán, 1972. (Citada como Colpocephalum subpachygaster)

Laemobothrion maximum (Scopoli, 1763)

FAMILIA: Laemobothriidae

HOSPEDADOR: Buteo buteo (L.) , Milvus milvus (L.), Circus aeruginosus (L.)

MATERIAL: 4 hembras, El Escorial, (Ceballos, leg.); 2 hembras, El Pardo, 9-VII1950 (B. de Quirós, leg.); 1 macho, Aranjuez, 12-IX-1950 (B.de Quirós), 2 hembras, Madrid, 5-II.1904 (Martínez de la Escalera,leg.) Colección MNCN

REFERENCIA: Martín, 1975.

Menacanthus pici (Denny, 1842)

FAMILIA: Menoponidae HOSPEDADOR : Picus viridis (L.)

MATERIAL: 2 machos, 2 ninfas, Madrid, II1921 (B. de Quirós, leg.) Colección MNCN REFERENCIA: Martín Mateo, 1984

Menacanthus eurysternus (Burmeister, 1838) FAMILIA: Menoponidae

HOSPEDADOR: Pica pica (L.)

MATERIAL: 1 hembra, Aranjuez (Las Infantas), X-1950 (B. de Quirós,leg.); 1 macho, 3 hembras, Pozuelo de Alarcón, I-1958 (Ruano, leg.). Colección MNCN

REFERENCIA: Martín y Jiménez Millán, 1967. (Citada como Menopon spiniferum)

Meromenopon meropis Clay \& Meinertzhagen, 1941

FAMILIA: Ancistronidae

HOSPEDADOR: Merops apiaster L.

MATERIAL: 1 macho, Bobadilla del Monte, 2-VIII-1975 (López Gordo,leg.); 1 hembra, Madrid, VII-1975 (López Gordo leg.) ; 2 hembras, Villaviciona de Odón (Martínez de la Escalera leg.) Colección MNCN REFERENCIA :Martín Mateo., 1977.

Meropoecus meropis Eichler, 1940

FAMILIA: Philopteridae

HOSPEDADOR : Merops apiaster L.

MATERIAL: 2 hembras, Madrid (Fuencarral),

II-1921 (B. de Quirós leg.) Colección MNCN REFERENCIA: Martín Mateo, 1977.

Myrsidea picae (Linnaeus, 1758)

FAMILIA: Menoponidae

HOSPEDADOR: Pica pica (L.)

MATERIAL: 2 hembras, Madrid, 1904

(Martínez de la Escalera) Colección MNCN

REFERENCIA: Martín y Jiménez Millán, 1967.

Neophilopterus tricolor (Burmeister, 1838)

FAMILIA: Philopteridae

Hospedador: Ciconia nigra (L.)
MATERIAL: 3 machos, 8 hembras, 15 ninfas; Buitrago, 2000-2003 (Lanzarot. leg.). Colección MNCN.

REFERENCIA: Lanzarot et al., 2005.

Philopterus corvi (Linnaeus, 1758)

FAMILIA: Philopteridae

HOSPEDADOR: Corvus corax L.

MATERIAL: 1 hembra, Madrid, II-1921 (B. de Quirós). Colección MNCN

REFERENCIA: Martín y Jiménez Millán, 1967.

Philopterus necopinatus (Zlotorzycka, 1964)

FAMILIA: Philopteridae

HOSPEDADOR: Acrocephalus schoenobaenus (L.)

MATERIAL: 1 hembra, Madrid, X-1923. Colección MNCN

REFERENCIA: Martín y Jiménez Millán, 1967. (Citada como Philopterus communis)

Philopterus pallescens (Denny, 1842)

FAMILIA: Philopteridae

HSPEDADOR: Parus major L.

MATERIAL: 3 hembras, Cercedilla (El Ventorrillo), III-1936 (J.Hernández leg.). Colección MNCN

REFERENCIA: Martín y Jiménez Millán, 1967. (Citada como Philopterus suflavescens)

Philopterus picae (Denny, 1842)

FAMILIA: Philopteridae

HOSPEDADOR: Pica pica (L.)

MATERIAL: 2 hembras, Madrid (Martínez de la Escalera leg.) Colección MNCN

REFERENCIA: Martín y Jiménez Millán, 1967. (Citada como Philopterus crassipes)

Philopterus turdi (Denny, 1842)

FAMILIA: Philopteridae

HOSPEDADOR: Turdus sp.

MATERIAL: 1 hembra, 4 ninfas, Pozuelo de Alarcon, III-1958 ( Ruano leg.) Colección MNCN

REFERENCIA: Martín y Jiménez Millán, 1967.

Quadraceps punctatus (Burmeister, 1838)

FAMILIA: Rallicolidae

HOSPEDADOR: Larus sp.

MATERIAL: 1 macho, Madrid, XII- 1921

(Martínez de la Escalera leg.). Colección MNCN

REFERENCIA: Martín y Jiménez Millán, 1967. (Citada como Degeeriella punctata)

Ricinus elongatus (Olfers, 1816)

FAMILIA: Ricinidae

HOSPEDADOR: Turdus merula L.

MATERIAL: 6 hembras, Cercedilla (El Ventorrillo), III-1936 (J. Hernández leg.); 4 hembras, Pozuelo de Alarcón, III-1958 (Ruano leg.). Colección MNCN

REFERENCIA: Martín Mateo, 1977.
Saemundssonia (Saemundssonia) lari (O. Fabricius, 1780)

FAMILIA: Rallicolidae

HOSPEDADOR: Larus ridibundus $\mathrm{L}$.

MATERIAL: 1 hembra, Madrid, XII-1904 (Martínez de la Escalera leg.) Colección MNCN

REFERENCIA: Martín Mateo, 1989.

Strigiphilus cursitans (Nitzsch, 1861)

FAMILIA: Philopteridae

HOSPEDADOR: Athene noctua (Scopoli)

MATERIAL: 1 hembra, 1 ninfa, Madrid (Martínez de la Escalera leg.) Colección $\mathrm{MNCN}$

REFERENCIA: Martín y Jiménez Millán, 1967. (Citada como Philopterus platystomus)

Strigiphilus cursor (Burmeister, 1838)

FAMILIA: Philopteridae

HOSPEDADOR: Asio flammeus (Pontopp.), Bubo bubo (L.)

MATERIAL: 4 machos, 1 hembra, Madrid (Villaverde), XII-1954 (Ruano leg.); 2 hembras, Montejo, IX-1950 (B. de Quirós leg.) Colección MNCN

REFERENCIA: Martín y Jiménez Millán, 1967. (Citada como Philopterus cursor)

Strigiphilus goniodicerus Eichler, 1949

FAMILIA: Philopteridae

HOSPEDADOR: Bubo bubo (L.), Bubo bubo hispanus Roths y Hartert

MATERIAL: 2 machos, 2 hembras, Brunete (Benedito leg.); 1 macho, 1 hembra, Madrid, XI-1904 (Benedito leg.). Colección MNCN

REFERENCIA: Martín y Jiménez Millán, 1967. (Citada como Philopterus crenulatus)

Upupicola upupae (Schrank, 1803)

FAMILIA: Degeeriellidae

HOSPEDADOR: Upupa epops L.

MATERIAL: 2 machos, Bobadilla del Monte, 4-VIII-1975; 2 machos, 3 hembras, El Pardo, 22-III-1934 (B. de Quirós leg.)

REFERENCIA: Martín Mateo, 1978.

MAMÍFEROS

Felicola genettae (Fresca, 1924)

FAMILIA: Trichodectidae

HOSPEDADOR: Genetta genetta (1.)

MATERIAL: 2 hembras, La Cabrera, I-1924 . Colección MNCN

REFERENCIA: Martín y Jiménez Millán, 1967. (Citada como Eutrichophilus genettae)

Trichodectes melis (J.C. Fabricius, 1805)

FAMILIA: Trichodectidae

HOSPEDADOR: Meles meles (L.)

MATERIAL: 1 macho, El Pardo. Colección MNCN

REFERENCIA: Martín y Jiménez Millán, 1967. 